exposure of the intrinsic muscles of the foot at their origin from the tubercle of the os calcis can be obtained.

The prognosis of cases dealt with surgically are good, provided: there are no mental defects, there is good control of the excreta, fits, if existent, can be dealt with and controlled by drugs, and lastly, that athetosis is not marked.

\title{
(3) Friedreich's Ataxia.
}

In the majority of cases in which ataxia and fits are associated patients can be taught to walk and become less ataxic with fewer fits if the foregoing operations are carried out.

The teaching of the older surgeons was that patients with fits and athetosis were totally unsuitable for surgical intervention. The modern view is that provided the fits can be controlled surgical intervention is of great value, the child's mind developing as the result of being able to walk.

\section{(4). Pseudo-hypertrophic Muscular Paralysis.}

The prognosis in such cases is exceedingly unfavourable. The classical picture of such a paralysis is well known. The calves are fixed and abnormally large and hard. The child finds it difficult to walk, the knees are contracted, and there is an equinus deformity of the foot with a scuttling gait, and for this reason the orthopædic surgeon's help is often required. A simple subcutaneous tenotomy of the tendo Achillis will often enable the patient to walk-particularly if after the tenotomy the lower limbs are placed in Thomas' splints for some four to six weeks, during which time the knees are carefully and firmly bandaged into position of dead extension.

Should deformities be more marked more radical operations are necessary similar to those already described.

Surgical treatment is of great value. It cannot be emphasized too st:ongly that simple operations can often enable the patient to continue to walk and get about for a long time, before he becomes bedridden and succumbs to the disease at the age of early adolescence.

\section{A CASE OF LOW BLOOD-PRESSURE TREATED WITH PITUITARY WHOLE GLAND BY THE MOUTH.}

\author{
BY BERNARD MYERS, C.M.G., M.D.
}

ARTERIaL blood-pressure is dependent upon four factors: the contracting force of the left ventricle, the volume of blood propelled by the contraction into the full arteries, the elasticity of which the middle coats of the large arteries are capable, and the peripheral resistance.

The systolic blood-pressure is synchronous with the systole of the ventricle and the diastolic with the diastole. By subtracting the diastolic from the systolic blood-pressure we obtain the figure representing the pulse-pressure. The figures obtained are far from being absolutely fixed ones, as the same individual may show quite definite changes during the twenty-four hours or even in a few minutes if subjected to stress or excitement, or again, on the other hand, during sleep. It is a good plan to take the blood-pressure at least an hour after exercise and before a meal. 
Among the causes of low blood-pressure F. W. Price mentions fatty myocardial degeneration, some cases of chronic interstitial myocarditis, most cases of chronic valvular disease, especially mitral and acute cardiac disease not accompanied by pronounced cyanosis. Other causes given by him are Addison's disease, early Graves' disease, congenital deficiency of vasomotor system, acute infective diseases, autointoxication (teeth, tonsils, \&c.), pulmonary tuberculosis, gastric disorders, diarrhœa, shock and collapse, severe bleeding, wasting diseases as cancer, neurasthenia, cerebral thrombosis, and excessive use of tobacco. He also mentions prolonged rest in the recumbent position and certain cases of very slow pulse.

Price is of opinion that a subnormal blood-pressure is more often an indication of derangement of vasomotor centre than of cardiac impairment.

Endocrine insufficiency is thought by some observers to play a part, and perhaps an important one, in the production of low blood-pressure, and the suprarenal, pituitary and thyroid glands have been suggested as the responsible causative factor in certain cases.

Of symptoms there may be mentioned asthenia, giddiness, faintness (more particularly of postural change), easy exhaustion, insomnia, mental depression, netirasthenia, irritable temper, subnormal temperature, sensitiveness to cold, pale or blue fingers, $\&$ c.

I quote the following case: A middle-aged man, who had been engaged in heavy work in the garden before dinner and which he continued after his meal, suddenly fainted. He had always previously been healthy and fit and came from a strong and healthy family.

As a youth he was an athlete and had won several hundred yards sprints during his career, but had always experienced a tendency to severe dyspnœa and exhaustion on running a 220 yards, 440 yards race, or any longer distance. $\mathrm{He}$ was fond of swimming, but if the water happened to be cold or very rough he felt breathless and faint and had to get out as quickly as he could. As a youth he had been subject to chilblains. He came from a family in which most of the members suffered from low blood-pressure, but were all fairly good athletes.

During the first faint he was unconscious for about twenty minutes, but with rest in bed for a few days and the exhibition of nux vomica and digitalis he soon made a good recovery. The digitalis, however, even when given in moderate doses, made his heart throb so severely that it had to be stopped.

After recovery his blood-pressure was found to vary between Ioo and 105 systolic and 78 to 80 diastolic. When the systolic pressure fell to 95 he always felt faint and weak. On one occasion it fell to 92.

The second faint took place in the Austrian Tyrol after a trying day, first in a hot train which ran up to the highlands, followed by a long walk along the dusty shores of the lake, and finally descent to the lower, now cold, levels again, where he had to wait an hour for a train and without food. In this instance he was unconscious for about ten minutes, but again made a complete and uninterrupted recovery.

The third faint occurred while bending down to put in some plants in his garden one evening about dusk. Having learnt that the uncomfortable feeling in the epigastrium, together with a quickening of the pulse, the throbbing of the heart, giddiness, breathlessness, and a feeling of not being sure of himself meant an imminent faint, he desisted from his work at once and was consequently only semiconscious, and not unconscious. 
A slowing of the pulse to 58 , or a quickening to over 80 per minute, were both danger signals, also mental and physical fatigue.

He kept well for three or four years, but during last summer while playing a game of croquet on a particularly hot day; he felt the epigastric symptoms, giddiness, and a fast pulse coming on, so he rested in the recumbent position for half an hour, after which he had practically recovered.

It is noteworthy that the day before the first faint he was able to run upstairs at a tube station, two steps at a time, without any inconvenience or symptoms.

Clinically, the heart and blood-vessels were normal, also the electrocardiogram and $\mathrm{X}$-ray of the heart.

With regard to treatment, compound tincture of cinchona, tincture of nux vomica and small doses of sodium bromide proved very useful and gave a feeling of fitness within twenty-four hours. Digitalis, even after only one or two doses, invariably caused marked palpitation and distress in the chest. Finally, pituitary whole gland was tried, first in $2 \mathrm{gr}$. doses, then in I gr., and lastly in $\frac{1}{2}$ gr. doses. Nothing gave such a feeling of security and freedom from symptoms of an impending faint as the pituitary whole gland (siccum). On one occasion, feeling very weak after influenza, he stayed at a country house for the week-end. He felt distinctly cheap even on the Monday morning and, being apprehensive of fainting, took I gr. of pituitary. In two hours' time all the symptoms had vanished, and the weakness had been replaced by a feeling of robustness, so that he could walk for several miles at an average pace without effort, and hold things easily in his hands which previously that same morning had seemed quite beyond his strength.

For three to four years he has taken $\frac{1}{2}$ gr. of pituitary whole gland at breakfast once or twice weekly. After a cold or influenza it is necessary to take the pituitary once or twice daily for a week, after which he reverts to twice weekly only. There can be no question whatsoever about the alleviation of his symptoms from taking pituitary and the feeling of security which it gives him. An experiment was made wherein no pituitary was taken for several weeks, when the blood-pressure figures were 102- 78 . After taking $\frac{1}{2} \mathrm{gr}$. of pituitary once daily for a week the figures were $110-78$, and continuing the treatment for a fortnight the systolic blood-pressure was 120 and the diastolic 78 .

It is a curious fact that so soon as the blood-pressure reached $120 \mathrm{~mm}$. Hg. systolic he suffered from throbbing in the head with a feeling of pressure and most of the symptoms of high blood-pressure. The figures which gave him the greatest comfort and the feeling of robustness was with a systolic of about II 5 and the diastolic of 78 .

No bad symptoms nor any inconvenience ever followed the taking of pituitary in the above doses, but 2 gr. three times a day when tried in one instance caused palpitation and had to be discontinued.

Of course one swallow does not make a summer, but in this instance a very definite case of low blood-pressure with distressing symptoms and a feeling of insecurity has, after treatment with pituitary whole gland by the mouth, been followed by freedom from symptoms and a feeling of robustness. 